\title{
DESCRIPCIÓN DE LOS RESULTADOS DE LA APLICACIÓN DE LA GUÍA DE MANEJO ODONTOLÓGICO PARA PACIENTES BAJO TERAPIA ANTICOAGULANTE EN CARDIODONTO, FUNDACION CARDIOVASCULAR DEL ORIENTE COLOMBIANO
}

\author{
${ }^{1}$ Uriel Mesa Herrera, ${ }^{2}$ Rubén Vargas, ${ }^{3}$ Luisa Fernanda Peñuela Balaguera, ${ }^{3}$ Rocío Del Pilar Sánchez Santos, \\ ${ }^{3}$ Sandra Carolina Rey Delgado, ${ }^{4}$ Anayancy Pérez Rivera. \\ ${ }^{1}$ Odontólogo, U.J., Fundación Cardiovascular del Oriente Colombiano. ${ }^{2} \mathrm{MD}$, Especialista en Medicina Interna . Fundación Cardiovascular del \\ Oriente Colombiano, ${ }^{3}$ Odontólogas, U. Santo Tomás, \\ ${ }^{4}$ Estudiante de X semestre, Facultad de Odontología, U. Santo Tomás \\ Autor responsable de correspondencia: Anayancy Pérez Rivera. \\ e-mail: anayancy@ustabuca.edu.co
}

\section{RESUMEN}

objetivo: Describir las complicaciones tromboembólicas y hemorrágicas derivadas de la aplicación de la guía de manejo odontológico del paciente anticoagulado, que se utiliza en el Departamento de Odontología de la Fundación Cardiovascular del Oriente Colombiano.

Materiales y Métodos: Mediante un estudio observacional descriptivo se revisaron las historias clínicas médicas y odontológicas de 108 individuos, con un promedio de edad de 54 años, 70\% del género masculino.

Resultados: La frecuencia más alta de cardiopatías fue la enfermedad coronaria (62.09\%) y las valvulopatías (40.74\%). En cuanto a la prescripción, previa intervención odontológica, la frecuencia para heparina, 50.92\% y warfarina 37.04\%. En relación con el protocolo se agruparon cuatro categorías en 2 grupos: alto riesgo de sangrado, que incluyó a las subcategorías: alto riesgo de tromboembolismo y bajo riesgo de tromboembolismo (72.22\%); bajo riesgo de sangrado con subcategorías: alto riesgo de tromboembolismo y bajo riesgo de tromboembolismo (27.78\%). Del total de la muestra, 13 pacientes (12.04\%) requirieron un agente local adicional a maniobras de presión y/o sutura para detener la hemorragia en la cavidad oral. Ningún paciente requirió administración de agentes parenterales. No se presentaron episodios tromboembólicos.

Conclusiones: Poseer dos ó más enfermedades cardiovasculares y haber requerido procedimientos odontológicos de alto riesgo de sangrado, confiere un incremento en el riesgo de sangrado posterior al procedimiento odontológico, factores que. se deben considerar para estudios posteriores. [Mesa U, Vargas R, Peñuela L, Sánchez R, Rey S, Pérez A. Descripción de los resultados de la aplicación de la guía de manejo odontológico para pacientes bajo terapia anticoagulante en Cardiodonto, Fundación Cardiovascular del Oriente Colombiano. Ustasalud 2003;1: 7-12].

PALABRAS CLAVES: Terapia anticoagulante, Cardiopatía, Tromboembolismo, Sangrado post-operatorio.

Results Description From a Guide for Dental Management of Anticoagulated Patients Used in the Dental Department of the Fundación Cardiovascular del Oriente Colombiano.

\begin{abstract}
Purpose: The purpose of this study was to describe the tromboembolic and bleeding complications from a guide for dental management of anticoagulated patients used in the Dental Department of the Fundacion Cardiovascular del Oriente Colombiano (FCV) where the patient is classified according to cardiogenic pathology.

Material and Methods: Dental and medical histories of 108 individuals were revised using a descriptive observational study, this in order to design the investigation instrument. The mean age group was 54 years old, $70 \%$ masculine.

Results: The highest prevalence of the cardio pathologies was for coronary disease (62.09\%) and valvular pathologies (40.74\%). The frequency for previous prescription dental intervention was heparin 50.92\% and warfarin, 37.04\%. According to the protocol, its four categories were subdivided in 2 groups: high risk of bleeding which included subcategories ARS and BRS (72.22\%). Low risk of bleeding with subcategories ART and BRT (27.78\%). Thirteen patients (12.04\%) required a local agent in addition to excessive pressure and/or sutures to detain bleeding. No patients required application of parenteral agents. No tromboembolic episodes were presented.

Conclusions: There were significant associations between some factors and postoperatory bleeding. Those associations will have to be consider for posterior studies.
\end{abstract}

KEY WORDS: Anticoagulated therapy, Cardio pathology, Tromboembolism, Post-operative bleeding. 


\section{INTRODUCCIÓN}

Los individuos que padecen ciertas dolencias cardíacas, tales como valvulopatías o enfermedad coronaria, que necesitan tratamiento de terapia anticoagulante con el fin de prevenir episodios, tromboembólicos, representan un problema especial en la consulta odontológica. La mayoría de los procedimientos odontológicos provocan algún tipo de sangrado; por lo tanto, el manejo de este tipo de pacientes obliga a valorar el riesgo del sangrado local y el riesgo de tromboembolismo, ya que estas dos variables pueden deteriorar la salud integral del paciente. ${ }^{1}$

Existe controversia sobre el manejo de los pacientes durante los procedimientos dentales. Muchos informes recomiendan descontinuar la terapia anticoagulante para llevar a cabo procedimientos quirúrgicos orales; otros sugieren mantener la terapia anticoagulante. Una combinación de warfarina-heparina se sugiere para pacientes con alto riesgo de tromboembolismo. En otros casos, se sugiere el uso de terapia antifribinolítica local (ácido tranexámico). ${ }^{2}$ Se han realizado evaluaciones aisladas para el manejo odontológico de estos pacientes, con el fin de evitar complicaciones hemorrágicas y/o tromboembólicas, derivadas del tratamiento odontológico. ${ }^{3}$

Desde 1998 la Fundación Cardiovascular del Oriente Colombiano (FCV), en su dependencia de Odontología (CARDIODONTO), ha definido una serie de recomendaciones que integran los conceptos descritos en la literatura respecto del tratamiento de pacientes anticoagulados. Clasifica al paciente de acuerdo con la cardiopatía en bajo 0 alto riesgo de tromboembolismo y el procedimiento odontológico como de mayor o menor riesgo de sangrado. El objetivo de este estudio es describir las complicaciones cardiovasculares y hemorrágicas en individuos bajo terapia anticoagulante, atendidos en CARDIODONTO, desde 1998 hasta 2001.

\section{MATERIALES Y MÉTODOS}

Se realizó un estudio de tipo observacional descriptivo, con el fin de describir los resultados de la aplicación de la guía de manejo odontológico en pacientes bajo terapia anticoagulante, atendidos en Cardiodonto (dependencia odontológica de la Fundación Cardiovascular del Oriente
Colombiano). La población estuvo conformada por individuos bajo terapia anticoagulante atendidos en Cardiodonto, desde agosto de 1998 hasta diciembre de 2001. Se tomó una muestra de conveniencia después de revisar un total de 201 historias clínicas, correspondientes a la totalidad de pacientes que asistieron a Cardiodonto en la fecha antes señalada; de ellas, 108 cumplieron los criterios de inclusión. Se incluyeron en este estudio todos los individuos que se encontraban bajo terapia anticoagulante (consumo de warfarina y heparina de alto y bajo peso molecular) que fueron atendidos en Cardiodonto y se les practicó algún tipo de procedimiento odontológico de alto o bajo riesgo de sangrado; pacientes con consumo de anticoagulantes por causa de otra condición sistémica, diferente a la profilaxis de episodios tromboembólicos, ya que ésta es la variable que condiciona una de las complicaciones que se describen. Las variables evaluadas en el estudio son: tromboembolismo y sangrado. Las diferencias entre las medias de las muestras de dos grupos se evaluaron mediante la prueba $t$ de student para variables continuas; cuando hubo más de dos grupos se utilizó el análisis de varianza ANOVA. Para hallar diferencias en la distribución de variables discretas, se aplicó la prueba Chi cuadrado. La base de datos se realizó en Epi Info 6.0 y el análisis de datos en Minitab 12.0. Todo el análisis consideró un nivel de significancia $\alpha=0.05$.

\section{RESULTADOS}

Se observó una edad promedio de 54.05 años \pm 16.56 y una frecuencia de $70.37 \%$ para el género masculino. La enfermedad coronaria y la valvulopatía fueron las más frecuentes con un $62.96 \%$ y $40.74 \%$, respectivamente; coexistían en un $10.19 \%$ de los pacientes. Se observó, adicionalmente, la coexistencia de dos ó más enfermedades cardiovasculares ( $\geq 2$ ) en un $20.37 \%$ de la muestra. La frecuencia de comorbilidad, asociada a la enfermedad cardiovascular fue de 64.81\%, de manera global, y la frecuencia de hipertensión arterial (HTA), la más alta con un $54.63 \%$ (Figura 1).

Categorizando la muestra, según la comorbilidad, se observó una relación dosis-respuesta entre la edad de los pacientes y la presencia de éstas, que fue estadísticamente significativa $44.71 \pm 18.74$ vs. $56.41 \pm 12.42$ vs. $64.29 \pm 12.02$ años $(\mathrm{p}<0.0001)$ para 0,1 ó $>1$ patologías coexistentes respectivamente (Figura 2).

8 


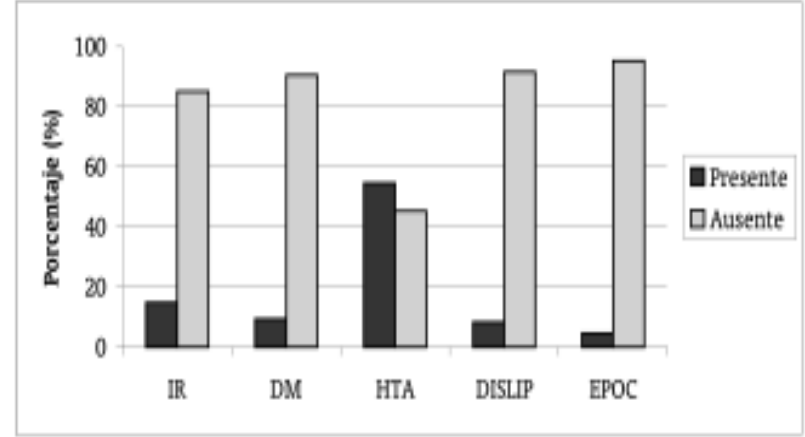

Figura 1. Frecuencia de patologías coexistentes - comorbilidad. IR: insuficiencia renal, DM: diabetes mellitus, HTA: hipertensión arterial, DISLIP: dislipidemia, EPOC: enfermedad pulmonar obstructiva crónica.

Se observó la prescripción de cinco (5) grandes grupos farmacológicos entre los cuales los nitratos (NITRATO) y los antihipertensivos (ANTIHTA) mostraron una frecuencia del $92.59 \%$ y $91.67 \%$ respectivamente. La prescripción previa intervención odontológica, para heparina 0 heparinas de bajo peso molecular, del 50.93\%, warfarina $37.04 \%$ y la asociación de ambas en un $12.04 \%$, pudiendo interpretarse esto último como un subgrupo transicional. Para antiagregantes plaquetarios representados principalmente por la aspirina (ASA), la frecuencia fue del $53.70 \%$, independientemente del anticoagulante 0 antiagregante.

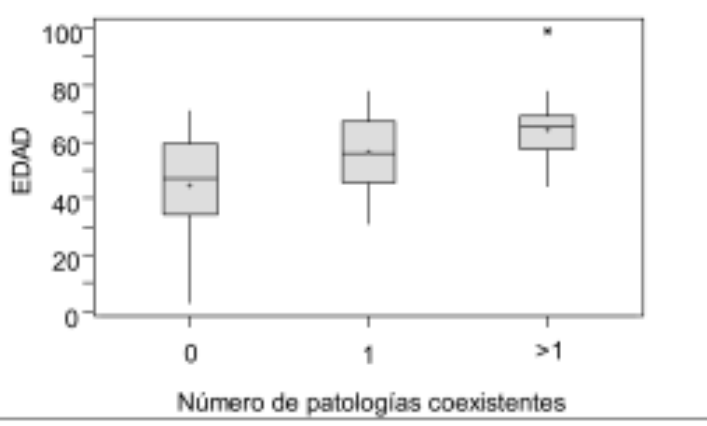

Figura 2. Diferencias en el promedio de edad, según el número de patologías coexistentes (comorbilidad).

Respecto de las variables fisiológicas y a las pruebas de coagulación sanguíneas, éstas fueron evaluadas el día previo al procedimiento ( $\mathrm{PRE}$ ), el día del procedimiento (PRO) y los días posteriores al mismo (POS). La variación en los signos vitales, considerados dentro del estudio, entre los períodos PRE, PRO y POS, se muestran en la Tabla 1. De ella se concluye que dichas variables fisiológicas no fueron alteradas en un nivel estadísticamente significativo, considerando los tres períodos previamente definidos (PRE PRO POS).

A diferencia del comportamiento estable en los signos vitales de los pacientes de estudio, los valores correspondientes a sus pruebas de coagulación sanguínea descendieron en concordancia con la aplicación del protocolo de riesgo odontológico tromboembólico, como era de esperarse (Tabla 1).

Tabla 1. Valores medios y diferencias de los signos vitales y las pruebas de coagulación durante el día previo al procedimiento odontológico (PRE), el día del procedimiento (PRO) y el posterior (POS).

\begin{tabular}{|c|c|c|c|c|c|}
\hline \multirow[b]{2}{*}{ Factor } & \multirow[b]{2}{*}{ PEEE } & \multirow[b]{2}{*}{ PRO } & \multicolumn{3}{|c|}{ Delta } \\
\hline & & & POS & PRO - PRE & (p) \\
\hline PAS & 121.9 & 120.9 & 119.8 & -1.0 & 0.760 \\
\hline PAD & 69.4 & 67.4 & 68.8 & 20 & 0.270 \\
\hline FC & 74.4 & 75.6 & 75.7 & 1.2 & 0.550 \\
\hline TEMP & 36.1 & 36.0 & 36.1 & 0.1 & 0.370 \\
\hline TrT & 52.1 & 39.4 & & -12.7 & 0.000 \\
\hline TP & 16.0 & 12.5 & & -2.5 & 0.000 \\
\hline INR & 1.58 & 1.22 & & -0.36 & 0.000 \\
\hline
\end{tabular}

AAS: presión arterial sistólica (mmHg), PaD: presión arterial disstólica (mmHg). FC- frecuencia cardiaca (1pm), TEMP. temperatura corporal CQ. TPE tiempo de tromboplestina partial (segundost. TP. tiempo de protrombine (sezundos) e INR: international nocmalized ratio. pi valor de probabalidad considerado estadisticamente signiflcativo menor de 0.05 
Tabla 2. Descripción de los procedimientos odontológicos según riesgo de sangrado.

\begin{tabular}{ccc}
\hline Procedimiento & $\begin{array}{c}\text { Alto riesgo } \\
\text { de sangrado }\end{array}$ & $\begin{array}{c}\text { Bajo riesgo } \\
\text { de sangrado }\end{array}$ \\
\hline Alisado radicular & $\mathrm{X}$ & \\
Raspaje campo abierto & $\mathrm{X}$ & \\
Exodoncia simple & $\mathrm{X}$ & \\
Exodoncia quirúrgica & $\mathrm{X}$ & \\
Regularización / rebordes & $\mathrm{X}$ & \\
Operatoria compleja & & $\mathrm{X}$ \\
Profilaxis & & $\mathrm{X}$ \\
Raspaje coronal & & $\mathrm{X}$ \\
\hline
\end{tabular}

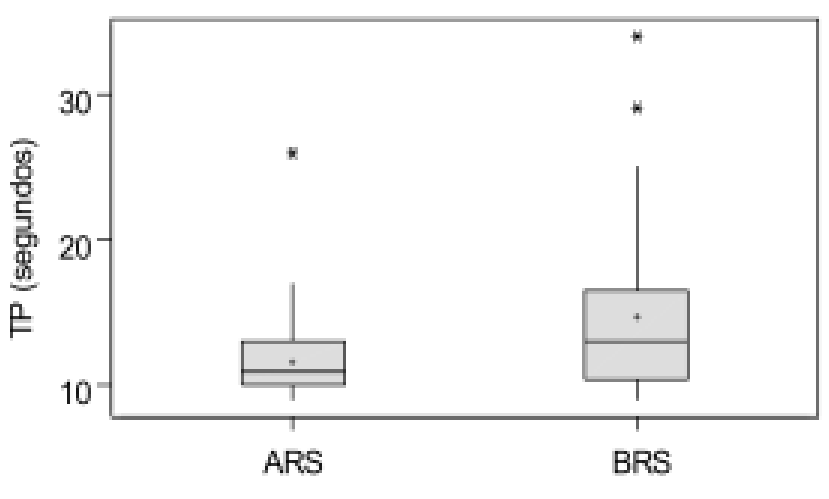

PROTOCOLO ASIGNADO

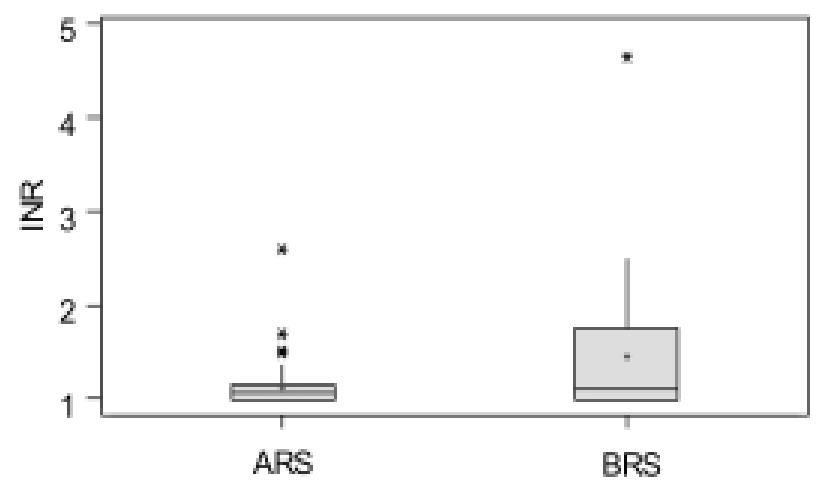

PROTOCOLO ASIGNADO

Figura 3. Diferencias entre medias de los valores del TP e INR en pacientes clasificados como ARS y BRS por el protocolo, durante el dia del procedimiento odontológico.
Las intervenciones odontológicas fueron agrupadas en dos categorías: alto riesgo de sangrado y bajo riesgo de sangrado (Tabla 2). Se observó que un $15 \%$ de pacientes debieron someterse a 4 ó 5 procedimientos simultáneamente y que la frecuencia de los clasificados como de procedimientos de alto riesgo de sangrado fue del $46.30 \%$, para el total de la muestra.

Se agruparon las cuatro categorías del protocolo en dos grupos, así: alto riesgo de sangrado (ARS), que incluyó las subcategorías alto/bajo riesgo de tromboembolismo (72.22\%), y bajo riesgo de sangrado (BRS), incluyendo, a su vez las dos subcategorías (27.78\%).

Los pacientes clasificados con ARS presentaron un tiempo de protrombina de $11.61 \pm 2.50$ vs. $14.72 \pm 5.86$ segundos $(\mathrm{p}<0.0001)$, y una Razón Normalizada Internacional (International Normalized Ratio) (INR) de $1.13 \pm 0.22$ vs. $1.46 \pm 0.75(\mathrm{p}=0.001)$ significativamente inferiores en relación con el grupo BRS el día del procedimiento odontológico (Figura 3), derivado de la transición del anticoagulante oral a heparina o la suspensión del primero, 48 horas antes del procedimiento.

Se observó que al grupo clasificado como ARS pertenecían pacientes de mayor edad $56.17 \pm 14.60$ vs. $48.53 \pm 20.05$ $(\mathrm{p}=0.013)$, lo cual estaría en relación directa con la mayor probabilidad de tener enfermedad cardiovascular (especialmente enfermedad coronaria y valvulopatía) al igual que comorbilidad.

Trece pacientes (12.04\%) del total de la muestra requirieron un agente local adicional a maniobras de presión y/0 sutura para detener la hemorragia en la cavidad oral.

Ningún paciente requirió administración de agentes parenterales y así mismo ningún otro presentó eventos tromboembólicos. Poseer dos ó más enfermedades cardiovasculares, y haber requerido procedimientos odontológicos de alto riesgo de sangrado, confiere un incremento en el riesgo de sangrado posterior al procedimiento odontológico (Tabla 3).

\section{DISCUSIÓN}

Este estudio hace un análisis de los resultados obtenidos de la aplicación de la guía de manejo odontológico para pacientes bajo terapia anticoagulante, implementada en Cardiodonto (dependencia odontológica de la Funda- 
Tabla 3. Evaluación de la asociación entre factores (considerados como exposición) y el riesgo de sangrado posterior a procedimientos odontológicos.

\begin{tabular}{lcccc}
\hline \multicolumn{1}{c}{ Factor } & \multicolumn{2}{c}{ Sangrado } & OR & p \\
& Positivo n:13 & Negativo n:95 & & \\
\hline Género maculino & $9(69.2)^{* *}$ & $67(70.5)^{* *}$ & 0.94 & 0.924 \\
ECV $\geq 2$ & $6(46.2)^{* *}$ & $16(16.8)^{* *}$ & 4.23 & 0.013 \\
Comorbilidad & $10(76.9)^{* *}$ & $60(63.2)^{* *}$ & 1.94 & 0.330 \\
Fármacos $>2$ & $5(38.5)^{* *}$ & $42(44.2)^{* *}$ & 0.76 & 0.695 \\
ARS & $13(100.0)^{* *}$ & $65(68.4)^{* *}$ & - & 0.017 \\
** Porcentaje & & & &
\end{tabular}

ción Cardiovascular del Oriente Colombiano), que se aplica desde 1998 con el objetivo de minimizar los riesgos tromboembólicos y aquellos relacionados con el sangrado posterior al procedimiento.

Los resultados sugieren que los pacientes que presentaron sangrado prolongado, posterior a la intervención odontológica, fueron aquellos a quienes se les realizó un procedimiento, que implicaba traumatismo de los tejidos. Por lo mismo, éstos fueron clasificados como de alto riesgo de sangrado. Hallazgos similares se encontraron en otro estudio, en el que los pacientes que presentaron sangrado prolongado fueron sometidos a procedimientos invasivos, como exodoncias y raspajes radículares, en presencia de enfermedad periodontal. Igualmente, en ninguno de los dos estudios se presentaron complicaciones tromboembólicas, aun cuando en el presente estudio se incluyeron pacientes de alto riesgo de tromboembolismo. ${ }^{2}$

Al igual que en el estudio realizado por Arteaga y col., no se tomaron en cuenta otros parámetros para medir el grado de inflamación y así poder diferenciar, de una forma más adecuada, este tipo de pacientes; por lo tanto, es preferible separar la muestra de acuerdo con el grado de inflamación puesto que éste influye en el grado de hemorragia. ${ }^{4}$

No se encontraron, en la literatura revisada, investigaciones que tuvieran en cuenta todos los procedimientos; en su gran mayoría correspondían a estudios relacionados con cirugía oral. Por lo tanto, los resultados no se pudieron comparar. Sin embargo, los rangos de seguridad, respecto del riesgo de hemorragia, demostraron que aumentaba el riesgo en el procedimiento odontológico al igual que en investigaciones anteriores. ${ }^{4-8}$

Dentro de los factores que mostraron asociación con sangrado post-operatorio se encuentran: la realización de procedimientos invasivos, la presencia de dos ó más cardiopatías, y la asociación de patologías cardiacas, concomitante con otro tipo de patologías de base principalmente la hipertensión arterial (Tabla 3). Este es el primer estudio que se realiza con el fin de analizar los resultados de la aplicación de la guía de manejo odontológico para pacientes bajo terapia anticoagulante, utilizada por la Fundación Cardiovascular del Oriente Colombiano en su dependencia CARDIODONTO; va encaminada a generar nuevas investigaciones, que profundicen los hallazgos obtenidos a partir de éste, para dar validez científica a dicha guía.

La muestra de este estudio fue tomada por conveniencia conformada por 108 individuos, lo que constituyó una limitante para establecer comparaciones entre varios grupos de tratamiento. A pesar de esto, se encontraron asociaciones significativas entre algunos factores y sangrado post-operatorio, que se deben considerar en posteriores estudios. 


\title{
BIBLIOGRAFÍA
}

1. Volker, H. El paciente con riesgo cardíaco en la clínica odontológica: un estudio controlado sobre las repercusiones en el sistema cardiovascular durante el tratamiento odontológico. Quintessence Odontología 1991;4:132-142.

2. Saour JN, Ali HA, Mammo LA, Sheik JO. Dental procedures in patients receiving oral anticoagulation therapy. J Heart Valve Dis. 1994;3:315-7.

3. Campbell JH, Alvarado F, Murray RA. Anticoagulation and minor oral surgery: should the anticoagulation regimen be altered? J Oral Maxillofac Surg. 2000;58:131-5; discussion 135-6.

4. Arteaga M. Uso de ácido tranexámico en pacientes anticoagulados durante cirugía oral. Universitas Odontológica 1998; 18:105-110.

5. Gaspar R. Empleo de colutorios de ácido tranexámico para prevenir la hemorragia postoperatoria anticoagulante. Quintessence 1998; 11:255-259.

6. Ramstrom G, Sindet-Pedersen S, may G, Blomback M, Alander U. Prevention of postsurgical bleeding in oral surgery using tranexamic acid without dose modification of oral anticoagulants. J Oral Maxillofac Surg. 1993;51:1211-6.

7. Bodner L, Weinstein JM, Baumgarten AK. Efficacy of fibrin sealant in patients on various levels of oral anticoagulant undergoing oral surgery. Oral Surg Oral Med Oral Pathol Oral Radiol Endod. 1998;86:421-4.

8. Souto JC, Oliver A, Zuazu-Jausoro I, Vives A, Fontcuberta J. Oral surgery in anticoagulated patients without reducing the dose of oral anticoagulant: a prospective randomized study. J Oral Maxillofac Surg. 1996;54:27-32; discussion 323

\section{CRÉDITOS}

\author{
Fundación Cardiovascular del Oriente Colombiano \\ Cardiodonto (FCV) \\ Clínica de Anticoagulación (FCV)
}

